\title{
25 Research Soure \\ Efficacy and Safety of Traditional Chinese Medicine Combined With First Lines Chemotherapy for Small- cell Lung Cancer: a Prospective Cohort Study
}

\section{Shuntai Chen}

China Academy of Chinese Medical Sciences Guanganmen Hospital

Jie He

Beijing University of Chinese Medicine

Shulin He

Beijing University of Chinese Medicine

Conghuang Li

China Academy of Chinese Medical Sciences Guanganmen Hospital

\section{Liang Geng}

Henan Cancer Hospital

Mingxi Sun

Beijing Nanyuan Hospital

Honggang Zheng

China Academy of Chinese Medical Sciences Guanganmen Hospital

Baojin Hua ( $\square$ huabaojinxs@126.com )

China Academy of Chinese Medical Sciences Guanganmen Hospital https://orcid.org/0000-00026927-7492

Original Article - Clinical Oncology

Keywords: OS, PFS, AEs, ORR, DCR, SCLC, TCM

Posted Date: February 15th, 2021

DOl: https://doi.org/10.21203/rs.3.rs-201637/v1

License: (c) (i) This work is licensed under a Creative Commons Attribution 4.0 International License.

Read Full License 


\section{Abstract}

Background: Small cell lung cancer (SCLC) tends to respond well to the standard treatment of chemotherapy, but disease progression, chemotherapy resistance, and adverse reactions still remain major problems that have long limited its effectiveness. Although Traditional Chinese Medicine (TCM) is wildly applied for patients with SCLC in China, using TCM in the treatment for SCLC is still in the absence of sufficient evidence.

Purpose: To evaluate the efficacy and safety of TCM combined with the first lines chemotherapy for SCLC.

Method: In this perspective, two-center cohort studies, overall survival (OS), progression free survival (PFS), overall response rate (ORR), and disease control rate (DCR) were compared between patients who received chemotherapy with or without TCM, with adverse events (AEs) also on the list of comparison.

Results: When the follow-up ended, 168 patients were included in this study. Compared with patients treated with chemotherapy alone (non-TCM group), those with TCM and chemotherapy (TCM group) had better PFS (adjusted HR 0.552, 95\% Cl 0.389-0.783, $P=0.01$ ), median PFS ( 11.83 months vs. 8.60 months, $P=0.004)$, rates of ORR ( $85.88 \%$ vs $75.90 \%$ after second cycle treatment, $61.2 \%$ vs. $43.4 \%$ after fourth cycle treatment), rates of DCR( $100 \%$ vs $98.80 \%$ after second cycle treatment, $95.3 \%$ vs. $89.2 \%$ after fourth cycle treatment). The number of cases with leukopenia, anorexia, constipation, fatigue, nausea in patients receiving TCM treatment is lower than those in patients not receiving TCM $(P<0.05)$. There was no difference between the two groups in median OS (23.4 months vs. 20.1 months, $P=0.301)$. Subgroup analysis showed there was no difference between the two groups in median OS with 34.5 months vs. 22.6 months for LS-SCLC $(P=0.586)$ and 18.5 months vs. 17.37 months for ES-SCLC $(P=0.162)$. Patients in the TCM group have better median PFS than patients in the non-TCM group for both LS-SCLC and ESSCLC with 14.73 months vs. 9.2 months $(P=0.040)$ and 9.53 months vs. 7.47 months $(P=0.007)$ respectively.

Conclusion: This study indicated that TCM combined with chemotherapy for SCLC may prolong PFS, improve therapeutic effect, reduce the side effects of chemotherapy, but may not be effective in the prolongation of OS. More large-scale and higher quality clinical studies are warranted to support our findings.

Clinical registration number: ChiCTR2000040151. (http://www.chictr.org.cn)

\section{Background}

Today, the world is still suffering from lung cancer, which is particularly true in China. In 2018, the number of newly confirmed lung cancer cases worldwide hit 2.09 million, of which the Chinese patients accounted for more than $700,000^{12}$. Also, lung cancer is the killer of most cancer deaths in China regardless of gender ${ }^{1}$. With an early metastatic spread to regional lymph nodes and distant sites and 
rapid growth, the aggressive disease of SCLC accounts for $13-15 \%$ of all lung cancer cases ${ }^{3}$. SCLC, taking its sensitivity into consideration, has been treated by chemotherapy as a standard method. At the same time, combining thoracic radiotherapy with chemotherapy has contributed to the survival rate of patients with limited-stage of SCLC ${ }^{4}$. However, most patients are usually sensitive to chemotherapy and relapse quickly with resistant diseases. The treatment and the patients' quality of life have thus been seriously affected. It is reported that within one year after the treatment, about $80 \%$ of patients with limited-stage small cell lung cancer (LS-SCLC) and almost all patients with extensive small cell lung cancer (ES-SCLC) relapse or progress, of which approximately $95 \%$ eventually die of disease progression $^{6}$.

Proven to be an effective way to treat multifarious diseases in Asia ${ }^{7}$, TCM has been widely used on SCLC patients during or after chemotherapy in China. According to some clinical trials, TCM plus chemotherapy could be an answer in boosting the performance of treatment. Meanwhile, TCM is also considered as an important adjuvant treatment for SCLC, which plays a positive role in reducing side effects of chemotherapy and improving patients' quality of life, but they are less convincing confined to their small sample sizes and poor quality ${ }^{8}$. Therefore, this prospective cohort study was conducted to evaluate the efficacy and safety of TCM combined with first lines chemotherapy for SCLC.

\section{Method}

This prospective cohort study was conducted in two centers in China (Guang'AnMen Hospital, China Academy of Chinese Medical Sciences, Henan Cancer Hospital) from October 1, 2018, to December 31, 2018. The study received ethical approval from the Guang'AnMen Hospital, China Academy of Chinese Medical Sciences (2018-083-KY-01). Informed consent was obtained from all patients. This study was registered in the Chinese Clinical Trial Registry (http://www.chictr.org.cn; ChiCTR2000040151).

\subsection{Patients}

Eligible patients had documented and pathologically confirmed SCLC. Patients were required to be older than 18 years old, registering an Eastern Cooperative Oncology Group (ECOG) performance score $\leq 2$, with normal hematopoietic, hepatic, renal functions and at least three-month life expectancy. Exclusion criteria included patients with non-small cell lung cancer (NSCLC) and patients who received surgeries. Children, pregnant or lactating women, and psychiatric patients were also excluded.

\subsection{Study Design}

Patients received first lines chemotherapy according to the guidelines of National Comprehensive Cancer Network (NCCN) ${ }^{9}$. During the clinical visits, related data were collected by trained doctors who then completed case report forms covering TCM therapy use and clinical outcomes. All enrolled patients 
received follow-ups with clinical visits after every cycle of chemotherapy and every three months after treatment. For patients who were not able for clinical visits, trained doctors conducted telephone followups. The follow-ups were completed when patients passed away, and for those alive, the follow-ups were done by December 31, 2019.

\subsection{Exposure}

Based on the Pharmacopoeia of the People's Republic of China $2015^{10}$, we defined exposure to TCM as a patient who received herbal decoction treatment prescribed by a TCM physician during and after chemotherapy for at least 6 months (TCM group). Depending on whether exposed to TCM or not, patients were placed into TCM group or non-TCM group.

\subsection{Outcome Measure}

The primary outcome measures were OS and PFS, which were defined as the interval from the first chemotherapy to the date of death and progression disease (PD) respectively. The secondary efficacy parameter was tumor responses: ORR and DCR, which were evaluated by referencing the initial CT findings used to define tumor development every two cycles of chemotherapy according to the Response Evaluation Criteria in Solid Tumor (version1.1) ${ }^{11}$. ORR included complete response (CR) and partial response (PR). DCR included CR, PR and stable disease (SD). AEs were evaluated by the National Cancer Institute Common Toxicity Criteria (NCl-CTC) 4.0. All outcome data were collected with case report forms (CRF).

\subsection{Statistical Analysis}

We evaluated differences in the distribution of baseline characteristics between patients who did or did not receive TCM therapy. Regarding other outcomes, the T-test or the Wilcoxon rank-sum test was used to evaluate data when appropriate. The Chi-Square test or Fisher's exact test was applied to the categorical data. And we used the Wilcoxon rank-sum test for the data with hierarchy.

The Kaplan-Meier survival curves were used to compare differences in OS and PFS between the TCM and non-TCM groups. The log-rank test was performed to compare the difference between the survival curves of the two groups. Subgroup analysis stratified by different stages was performed. The Cox proportional hazard model was used to assess the prognostic values of variables.

All statistical analyses were performed using SPSS software version 26 (SPSS Inc., Chicago, IL, USA) and Graphpad Prism 8 (GraphPad Software Inc., La Jolla, CA, USA). All tests were two-sided, with the statistical significance level set at .05 . 


\section{Result}

\subsection{Characteristics of Study Participants}

Between October 2013 and December 2018, 190 patients participated in this study, among which 23 were excluded as 3 were confirmed with other tumors, 3 received surgeries, 1 had no definite stage, 5 had no complete clinical data, 4 were in the middle of treatment and 4 exited, leaving the final number of patients eligible for analysis to be 168 (Figure. 1). The lost 12 patients had finished 4-6 cycles of chemotherapy and were follow-up by at least 1 time, making them be evaluated as censored data. Among the final participants, 86 (46.2\%) were placed in the TCM group and 86 (53.8\%) were in the non-TCM group. No statistically significant difference existed between the two groups in terms of age, sex, disease stage at initial diagnosis, ECOG performance score at baseline, pleural effusions at initial diagnosis, brain metastases at initial diagnosis, chemotherapy regimens, history of TRT, history of PCl, smoking history, family history (Table 1). 
Table 1

Patient demographics and baseline characteristics.

\begin{tabular}{|c|c|c|c|c|}
\hline & $\begin{array}{l}\text { TCM Group ( } \mathrm{n} \\
=85)\end{array}$ & $\begin{array}{l}\text { Non-TCM Group ( } \mathrm{n} \\
=83 \text { ) }\end{array}$ & $\begin{array}{l}\text { All patients }(n= \\
168)\end{array}$ & $\begin{array}{l}P \\
\text { value }\end{array}$ \\
\hline $\begin{array}{l}\text { Median age at baseline, y } \\
\text { (range) }\end{array}$ & $61(23,75)$ & $60(40,75)$ & $60(23,75)$ & 0.815 \\
\hline \multicolumn{5}{|l|}{ Sex } \\
\hline Male & $64(75.3)$ & $63(75.9)$ & $127(75.6)$ & 0.927 \\
\hline Female & $21(24.7)$ & $20(24.1)$ & $41(24.4)$ & \\
\hline \multicolumn{5}{|l|}{$\begin{array}{l}\text { Disease stage at initial } \\
\text { diagnosis }\end{array}$} \\
\hline Limited-stage & $45(52.9)$ & $52(62.7)$ & $97(57.7)$ & 0.203 \\
\hline Extensive-stage & $40(47.1)$ & 31 (37.3) & $71(42.3)$ & \\
\hline \multicolumn{5}{|l|}{$\begin{array}{l}\text { ECOG performance score at } \\
\text { baseline }\end{array}$} \\
\hline 0 & $1(1.2)$ & $1(1.2)$ & $2(1.2)$ & 1.000 \\
\hline 1 & $77(90.6)$ & $75(90.4)$ & $152(90.5)$ & \\
\hline 2 & $7(8.2)$ & $7(8.4)$ & $14(8.3)$ & \\
\hline \multicolumn{5}{|l|}{$\begin{array}{l}\text { Pleural effusions at initial } \\
\text { diagnosis }\end{array}$} \\
\hline Yes & $14(16.5)$ & $16(19.3)$ & $30(17.9)$ & 0.635 \\
\hline No & $71(83.5)$ & $67(80.7)$ & $138(82.1)$ & \\
\hline \multicolumn{5}{|l|}{$\begin{array}{l}\text { Brain metastases at initial } \\
\text { diagnosis }\end{array}$} \\
\hline Yes & $8(9.4)$ & $2(2.4)$ & $10(6.0)$ & 0.111 \\
\hline No & $77(90.6)$ & $81(97.6)$ & $158(94.0)$ & \\
\hline \multicolumn{5}{|l|}{ Chemotherapy regimens } \\
\hline EP & $46(54.1)$ & $53(63.9)$ & $99(58.9)$ & 0.144 \\
\hline EC & $36(42.4)$ & $24(28.9)$ & $60(35.7)$ & \\
\hline
\end{tabular}

NOTE: Data shown are $N(\%)$ unless otherwise indicated.

Abbreviations: ECOG, Eastern Cooperative Oncology Group; EP, Etoposide and cisplatin; EC, Etoposide and carboplatin; TRT, thoracic radiation therapy; PCl, Prophylactic Cranial Irradiation. ${ }^{a}$ Other chemotherapy regimens included Etoposide and lobaplatin/nedaplatin 


\begin{tabular}{|c|c|c|c|c|}
\hline & $\begin{array}{l}\text { TCM Group }(\mathrm{n} \\
=85)\end{array}$ & $\begin{array}{l}\text { Non-TCM Group (n } \\
=83 \text { ) }\end{array}$ & $\begin{array}{l}\text { All patients }(n= \\
168)\end{array}$ & $\begin{array}{l}P \\
\text { value }\end{array}$ \\
\hline Other ${ }^{\mathrm{a}}$ & $3(3.5)$ & $6(7.2)$ & $9(5.4)$ & \\
\hline \multicolumn{5}{|c|}{ History of TRT } \\
\hline Yes & $60(70.6)$ & $60(72.3)$ & $120(71.4)$ & 0.807 \\
\hline No & $25(29.4)$ & $23(27.7)$ & $48(28.6)$ & \\
\hline \multicolumn{5}{|c|}{ History of $\mathrm{PCl}$} \\
\hline Yes & $26(30.6)$ & $29(34.9)$ & $55(32.7)$ & 0.548 \\
\hline No & $59(69.4)$ & $54(65.1)$ & 113 (67.3) & \\
\hline \multicolumn{5}{|c|}{ Smoking history } \\
\hline Yes & $46(54.1)$ & 51 (61.4) & 97 (57.7) & 0.336 \\
\hline No & 39 (45.9) & 32 (38.6) & $71(42.3)$ & \\
\hline \multicolumn{5}{|c|}{ Family history } \\
\hline Yes & 15 (17.6) & 16 (19.3) & $31(18.5)$ & 0.785 \\
\hline no & $70(82.4)$ & 67 (80.7) & 137 (81.5) & \\
\hline \multicolumn{5}{|c|}{ NOTE: Data shown are $N(\%)$ unless otherwise indicated. } \\
\hline \multicolumn{5}{|c|}{$\begin{array}{l}\text { Abbreviations: ECOG, Eastern Cooperative Oncology Group; EP, Etoposide and cisplatin; EC, Etoposide } \\
\text { and carboplatin; TRT, thoracic radiation therapy; PCl, Prophylactic Cranial Irradiation. }{ }^{\mathrm{a} O t h e r} \\
\text { chemotherapy regimens included Etoposide and lobaplatin/nedaplatin }\end{array}$} \\
\hline
\end{tabular}

\subsection{OS and PFS}

OS and PFS were calculated to evaluate the impact of TCM therapy on prognosis. The median OS was 23.4 months in patients who received TCM therapy, whereas OS in patients without TCM therapy was 20.1 months ( $P=0.301$, Fig. 2). The median PFS registered 11.83 months and 8.60 months in the TCM and the non-TCM group respectively. There was a significant difference between the groups in PFS ( $P=$ 0.004 , Fig. 3). The use of Cox proportional hazards regression for confounder control yielded that TCM was a significant clinical factor for PFS (adjusted HR 0.552, 95\% $\mathrm{Cl} 0.389-0.783, P=0.01$, Fig. 4), but was not a significant clinical factor for OS compared with non-TCM $(P>0.05)$.

Subgroup analysis stratified by different stages was performed. For LS-SCLC, the median OS was 34.5 months in the TCM group, and that in the non-TCM group was 22.6 months ( $P=0.586$, Fig. 5$)$. The median PFS registered 14.73 months and 9.2 months in the two groups respectively $(P=0.040$, Fig. 6$)$. 
For ES-SCLC, the median OS for the TCM group was 18.5 months compared with 17.37 months for the non-TCM group ( $P=0.162$, Fig. 7$)$. The median PFS for patients in the TCM group was 9.53 months, whereas, for patients in the non-TCM group, the median PFS was 7.47 months $(P=0.007$, Fig. 8).

\subsection{ORR and DCR}

ORR and DCR were evaluated (Table 2). After two cycles of treatments, ORR of the TCM group recorded $85.88 \%$ compared with $75.90 \%$ of the non-TCM group. DCR of the TCM group was $100 \%$ whereas, for the non-TCM group, the percentage was $98.80 \%$. When four cycles of treatments were finished, ORR of the TCM group was $61.2 \%$ better than that of the non-TCM group (43.4\%). Meanwhile, DCR for the TCM group notched up $95.3 \%$ compared with $89.2 \%$ for the non-TCM group.

Table 2

ORR and DCR for SCLC with or without TCM

\begin{tabular}{|lllllll|}
\hline & CR & PR & SD & PD & ORR & DCR \\
\hline $\begin{array}{l}\text { After second cycle } \\
\text { treatment }\end{array}$ & & & & & & \\
\hline TCM & $2(2.4 \%)$ & $71(83.5 \%)$ & $12(14.1 \%)$ & $0(0 \%)$ & $73(85.88 \%)$ & $85(100 \%)$ \\
\hline Non-TCM & $0(0 \%)$ & $63(75.9 \%)$ & $19(22.9 \%)$ & $1(1.2 \%)$ & $63(75.90 \%)$ & $82(98.80 \%)$ \\
\hline $\begin{array}{l}\text { After fourth cycle } \\
\text { treatment }\end{array}$ & & & & & & \\
\hline TCM & $0(0 \%)$ & $52(61.2 \%)$ & $29(34.1 \%)$ & $4(4.7 \%)$ & $52(61.2 \%)$ & $81(95.3 \%)$ \\
\hline Non-TCM & $2(2.4 \%)$ & $34(41 \%)$ & $38(45.8 \%)$ & $9(10.8 \%)$ & $36(43.4 \%)$ & $74(89.2 \%)$ \\
\hline
\end{tabular}

\subsection{Adverse Events}

The number of cases with leukopenia, anorexia, constipation, fatigue, nausea in patients receiving TCM treatment is lower than that in patients without TCM $(P<0.05$, Table 3$)$. There was no difference between the two groups for other adverse events $(P>0.05$, Table 3$)$. 
Table 3

The number of cases with the highest adverse reactions during treatment

\begin{tabular}{|c|c|c|c|c|c|}
\hline & \multicolumn{2}{|l|}{ Grade $1 / 2$} & \multicolumn{2}{|c|}{ Grade 3/4 } & \multirow[t]{2}{*}{$P$} \\
\hline & TCM & Non-TCM & TCM & Non-TCM & \\
\hline \multicolumn{6}{|l|}{ Hematologic } \\
\hline Anemia & $40(47.1 \%)$ & $50(60.2 \%)$ & $2(2.4 \%)$ & $6(7.2)$ & 0.032 \\
\hline Leukopenia & $59(69.4 \%)$ & $48(57.8 \%)$ & $6(7.1 \%)$ & $20(24.1)$ & $0.009 *$ \\
\hline Neutropenia & $44(51.8 \%)$ & $31(37.3 \%)$ & $7(8.2 \%)$ & 13(15.7) & 0.112 \\
\hline Thrombocytopenia & $7(8.2 \%)$ & $8(9.6 \%)$ & $0(0)$ & $2(2.4)$ & 0.482 \\
\hline \multicolumn{6}{|l|}{ Nonhematologic } \\
\hline ALT increase & $3(3.5 \%)$ & $1(2.4 \%)$ & $0(0)$ & $0(0)$ & 0.630 \\
\hline AST increase & $2(2.4 \%)$ & $4(4.8 \%)$ & $0(0)$ & $0(0)$ & 0.656 \\
\hline ALP increase & $1(1.2 \%)$ & $1(1.2 \%)$ & $0(0)$ & $0(0)$ & 1.000 \\
\hline Tbil increase & $4(4.7 \%)$ & $2(2.4 \%)$ & $0(0)$ & $0(0)$ & 0.699 \\
\hline Cr increase & $0(0)$ & $5(6.0 \%)$ & $0(0)$ & $0(0)$ & 0.065 \\
\hline Anorexia & $49(57.6 \%)$ & $58(69.9 \%)$ & $1(1.2 \%)$ & $8(9.6 \%)$ & $0.002^{*}$ \\
\hline Constipation & $22(25.9 \%)$ & $32(38.6 \%)$ & $1(1.2 \%)$ & $8(9.6 \%)$ & $0.004^{\star}$ \\
\hline Fatigue & $50(58.8 \%)$ & $71(85.5 \%)$ & $2(2.4 \%)$ & $4(4.8 \%)$ & $0.000^{*}$ \\
\hline Nausea & $28(32.9 \%)$ & $50(60.1 \%)$ & $4(4.7 \%)$ & $7(8.4 \%)$ & $0.000^{*}$ \\
\hline Vomiting & $19(22.4 \%)$ & $25(30.1 \%)$ & $0(0)$ & $2(2.4 \%)$ & 0.150 \\
\hline$\star P<0.05$ & & & & & \\
\hline
\end{tabular}

\section{Discussion}

With the treatment of SCLC unchanged for years, the survival rate of the disease is one of the lowest among all cancer cases ${ }^{12}$. For greater survival benefits, TCM is widely used in SCLC as an adjuvant to chemotherapy in China ${ }^{8}$. To our knowledge, this is the first prospective cohort study to evaluate the efficacy and safety of TCM combining first lines chemotherapy for SCLC. Among the 168 patients who could be analyzed for efficacy, the results show that compared with chemotherapy alone, TCM plus chemotherapy can provide a relatively better prognosis, encouraging effect, and fewer AEs for SCLC.

Although patients who received TCM treatment had a better median OS (23.4 months vs 20.1 months), there is no significant difference between the two groups in OS according to survival analysis. Also, 
based on Cox proportional hazards regression, TCM was not a significant clinical factor for OS. We believe that the reasons for the above results may be caused by too much censored data and insufficient follow-up time. Therefore, we will continue to follow up to see whether the results will change. Besides, according to the adjusted Cox regression analysis, TCM is a protective clinical factor for PFS. Survival analysis also showed that patients who received TCM treatment had better PFS than patients who received chemotherapy alone. Subgroup analysis according to different stages was implemented as the prognosis of SCLC at different stages varies greatly. The subgroup analysis also yielded similar results that there was no significant difference in OS with or without TCM for LS-SCLC or ES-SCLC. In terms of PFS, it reported great differences between the two groups for both LS-SCLC and ES-SCLC. This study also yielded similar results to the meta-analysis we did before [8], which further demonstrated that the combination of TCM and chemotherapy can enhance the efficiency of treatment while reducing the side effects related to chemotherapy.

The strengths of this study are its size (>150 patients), multicenter prospective cohort study design, and the TCM exposure defined. Most TCM prescriptions mentioned in this study are not exactly the same. According to the theory of TCM, a personalized treatment plan should be adopted according to a patient's physical condition. Therefore, we were referring to the general concept of TCM, rather than a fixed formula or a certain TCM patent medicine.

There are indeed some limitations of this study. First, the follow-up was relatively short for some patients considering the funding constraints. Second, its findings are limited to the population studied: patients with ECOG score $\leq 2$, whose life expectancy is longer than three months. Only patients whose survival period is expected to be longer than three months were included since the aim of this study is to observe the effect of TCM combined with chemotherapy. If the survival period is too short, the efficacy of TCM cannot be fully observed. Hence, the results of this study cannot be generalized to patients with relatively poor physical conditions.

\section{Conclusion}

Based on the results of this study, we can conclude that TCM combined with first lines chemotherapy for SCLC may prolong PFS, improve therapeutic effect, and reduce the side effects of chemotherapy. More clinical studies with higher levels of evidence and more participants are expected to be warranted to support our findings.

\section{Abbreviations}

SCLC: Small Cell Lung Cancer; NSCLC: non-small cell lung cancer; LS-SCLC: Limited-Stage Small Cell Lung cancer; ES-SCLC: Extensive-Stage Small Cell Lung cancer; TCM: Traditional Chinese Medicine; ECOG: Eastern Cooperative Oncology Group; NCCN: National Comprehensive Cancer Network; OS: Overall Survival; PFS: Progression free survival; PD: progression disease; ORR: Overall response rate; DCR: disease control rate CR: complete response; PR: partial response; SD: stable disease; AEs: Adverse events; 
NCI-CTC: National Cancer Institute Common Toxicity Criteria; CRF: case report forms: EP: Etoposide and cisplatin; EC: Etoposide and carboplatin; TRT: thoracic radiation therapy; PCI, Prophylactic Cranial Irradiation.

\section{Declarations}

\section{Acknowledgments}

We want to express our sincere gratitude to all patients who participated in this study and their families. We also thank doctors of the Department of Oncology from Guang'anmen Hospital, China Academy of Chinese Medical Sciences, and Henan Cancer Hospital, who recruited patients. We thank Ms. Yi Chu for manuscript writing assistance. Finally, we would like to thank Guang'anmen Hospital, China Academy of Chinese Medical Sciences and National Science and Natural Science Foundation of China for their financial support.

\section{Author Contributions}

All authors contributed to the study conception and design. Material preparation, data collection and analysis were performed by Jie He, Shulin He, Conghuang Li, Liang Geng, Mingxi Sun, Honggang Zheng. The first draft of the manuscript was written by Shuntai Chen and all authors commented on previous versions of the manuscript. All authors read and approved the final manuscript.

\section{Funding}

This work was supported by Guang'AnMen Hospital, China Academy of Chinese Medical Sciences (grant number: 59902) and National Natural Science Foundation of China (grant number: 81673961). The funders had no role in the study design in the collection, analysis, and interpretation of data.

\section{Ethics declarations}

\section{Ethics approval and consent to participate}

This study was performed in line with the principles of the Declaration of Helsinki. Approval was granted by the Ethics Committee of Guang'AnMen Hospital (2018-083-KY-01). Informed consent was obtained from all individual participants included in the study.

\section{Consent for publication}

Written informed consent for publication was obtained from all participants.

\section{Competing interests}

The authors declare that the research was conducted in the absence of any commercial or financial relationships that could be construed as a potential conflict of interest. 


\section{Availability of data and material}

The data of patients involved in this study are available on request from the corresponding author Dr. Baojin Hua. The data are not publicly available due to patients' privacy

\section{References}

1. Chen W, Zheng R, Zeng H, Zhang S. Epidemiology of lung cancer in $\mathrm{C}$ hina. Thoracic cancer. 2015;6(2):209-215. https ://doi.org/10.1111/1759-7714.12169

2. Hong QY, Wu G-M, Qian GS, et al. Prevention and management of lung cancer in China. Cancer. 2015;121(S17):3080-3088. https ://doi.org/10.1002/cncr.29584

3. Shi Y-X, Sheng D-Q, Cheng L, Song X-Y. Current Landscape of Epigenetics in Lung Cancer: Focus on the Mechanism and Application. Journal of Oncology. 2019;2019. https ://doi.org/10.1155/2019/81073 18

4. Kalemkerian GP, Schneider BJ. Advances in small cell lung cancer. Hematology/Oncology Clinics. 2017;31(1):143-156. https ://doi.org/10.1016/j.hoc.2016.08.005.Review

5. Waqar SN, Morgensztern D. Treatment advances in small cell lung cancer (SCLC). Pharmacology \& therapeutics. 2017;180:16-23. https ://doi.org/10.1016/j.pharmthera .2017.06.002

6. Dazzi C, Cariello A, Casanova C, et al. Gemcitabine and paclitaxel combination as second-line chemotherapy in patients with small-cell lung cancer: a phase II study. Clinical Lung Cancer. 2013;14(1):28-33. https ://doi.org/10.1016/j.cllc.2012.03.003

7. Lv S, Ding Y, Zhao H, Liu S, Zhang J, Wang J. Therapeutic potential and effective components of the Chinese herb Gardeniae fructus in the treatment of senile disease. Aging and disease. 2018;9(6):1153. https ://doi.org/10.14336/AD.2018.0112

8. Chen S, Bao Y, Xu J, et al. Efficacy and safety of TCM combined with chemotherapy for SCLC: a systematic review and meta-analysis. Journal of Cancer Research and Clinical Oncology. 2020:1-23. https ://doi.org/10.1007/s00432-020-03353-0

9. Kalemkerian GP, Loo BW, Akerley W, et al. NCCN guidelines insights: small cell lung cancer, version 2.2018. Journal of the National Comprehensive Cancer Network. 2018;16(10):1171-1182. https ://doi.org/10.6004/jnccn.2018.0079.

10. Commission CP. Pharmacopoeia of the People's Republic of China; Chinese Medical Science and Technology Press: Beijing, 2015; Vol. I.255-258.

11. Nishino M, Jagannathan JP, Ramaiya NH, Van den Abbeele AD. Revised RECIST guideline version 1.1: what oncologists want to know and what radiologists need to know. American Journal of Roentgenology. 2010;195(2):281-289. https ://doi.org/10.2214/AJR.09.4110

12. Thomas A, Vilimas R, Trindade $C$, et al. Durvalumab in combination with olaparib in patients with relapsed SCLC: results from a phase II study. Journal of Thoracic Oncology. 2019;14(8):1447-1457. https ://doi.org/10.1016/j.jtho.2019.04.026 
Figures

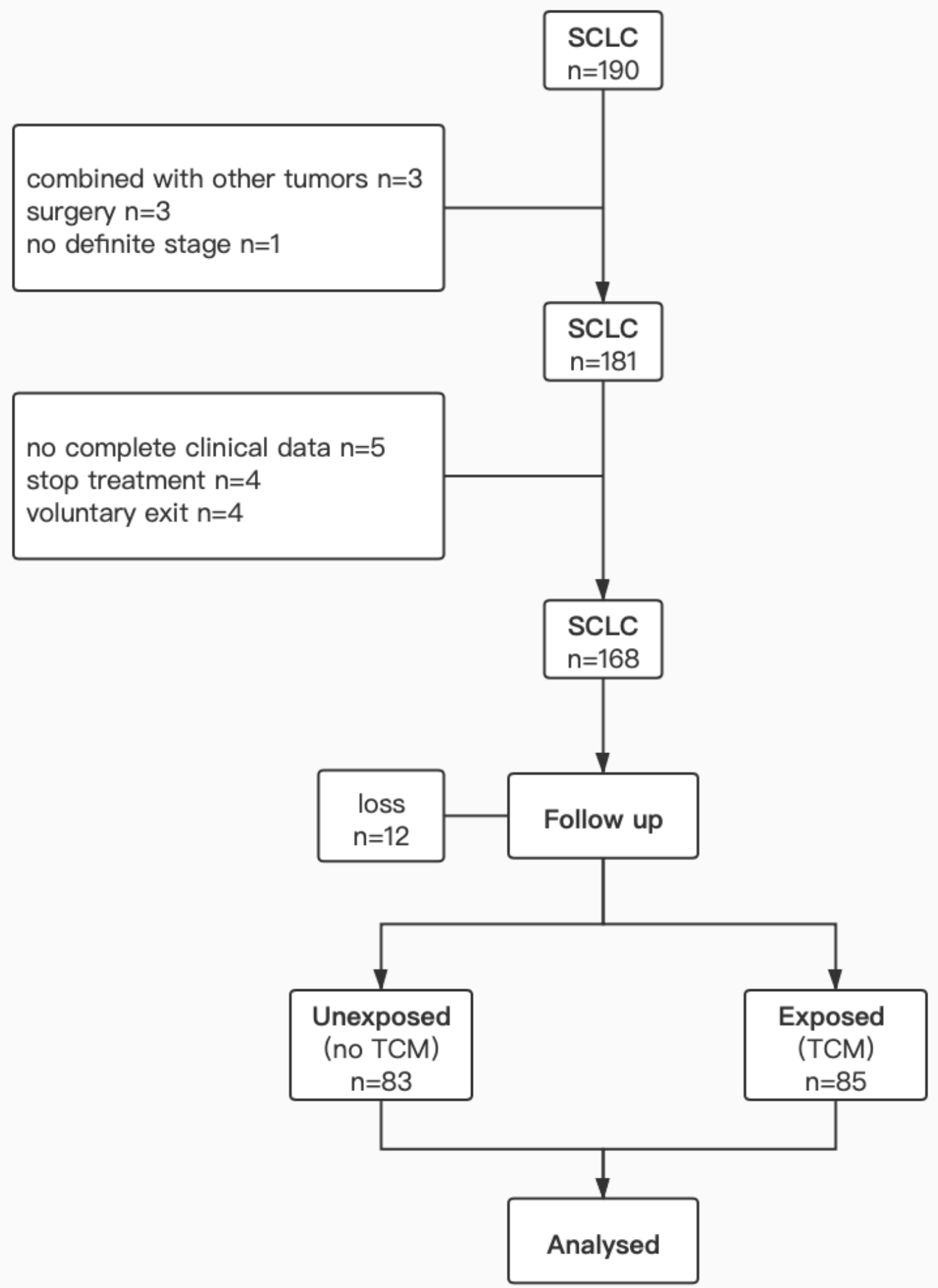

Figure 1

Flow Diagram 


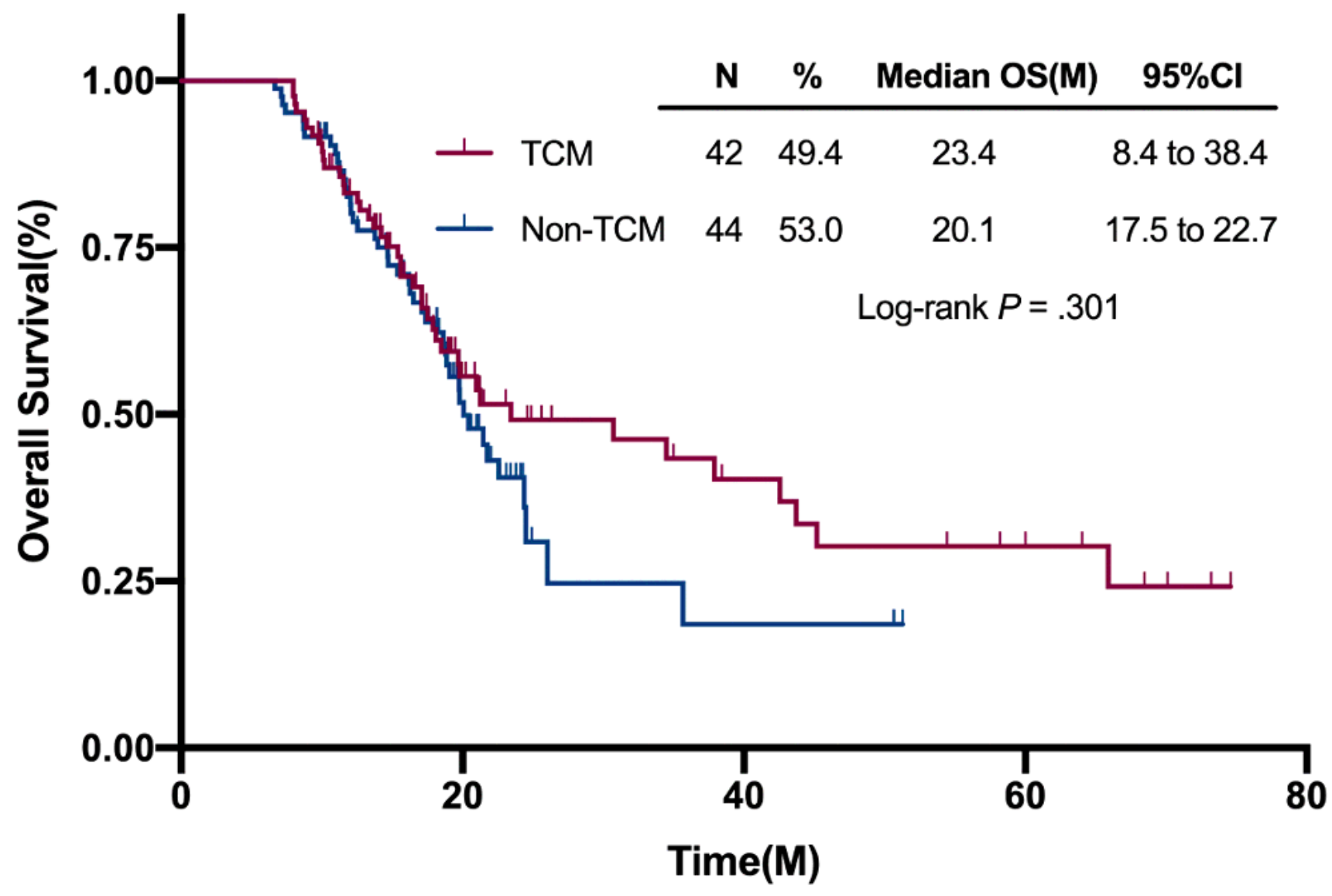

Figure 2

Survival curves of TCM for SCLC in OS 


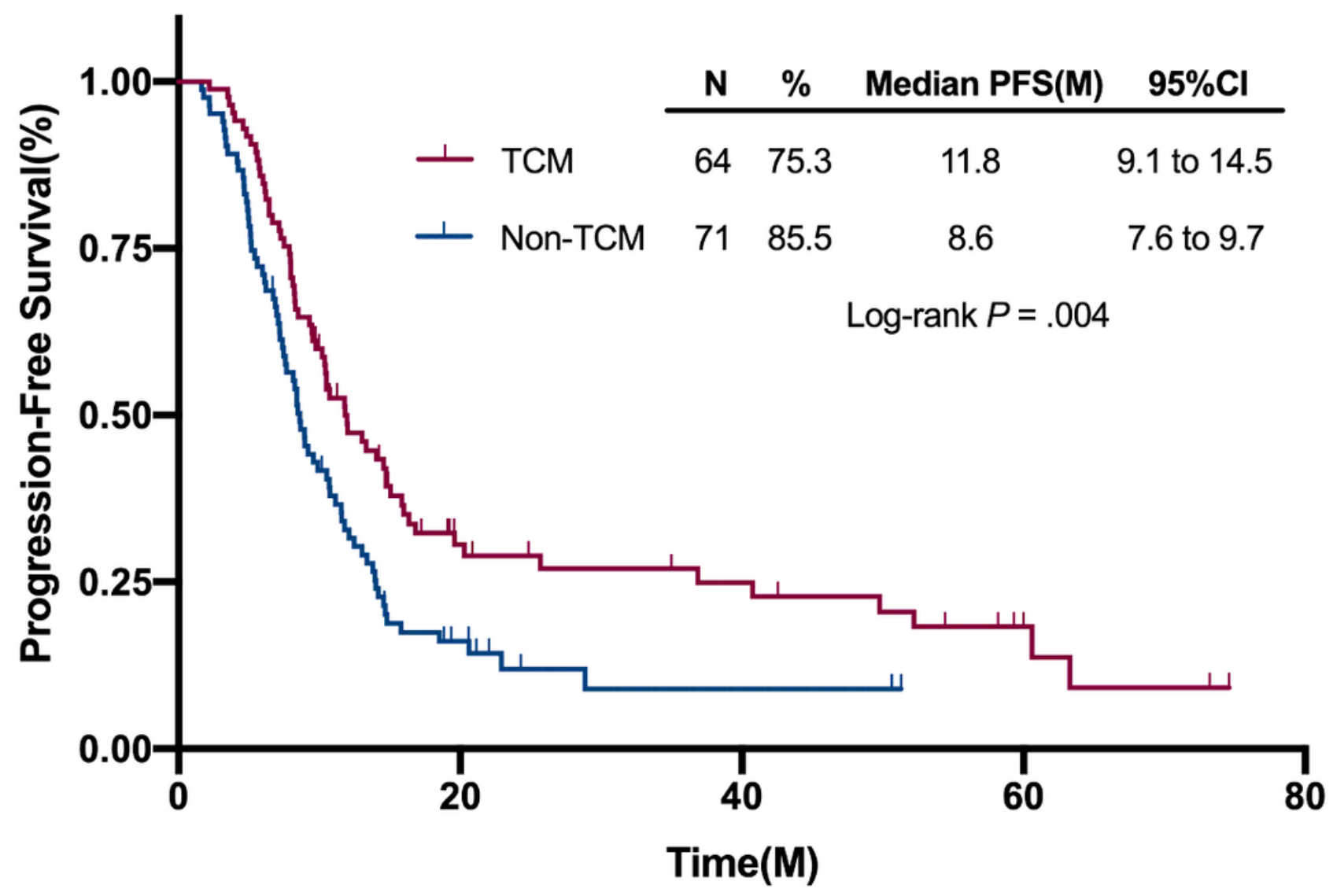

Figure 3

Survival curves of TCM for SCLC in PFS 


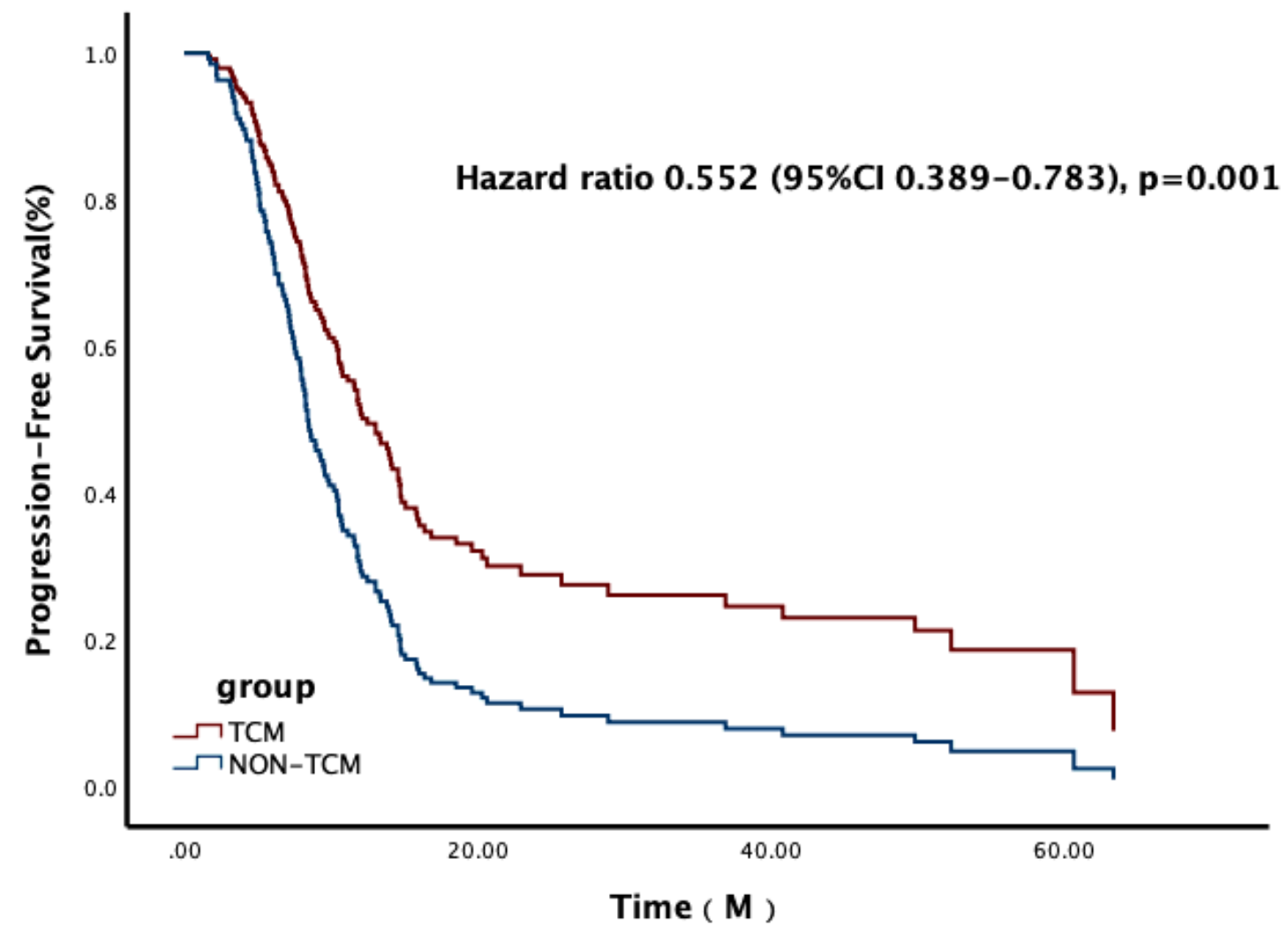

Figure 4

The use of Cox proportional hazards regression for confounder control yielded that TCM was a significant clinical factor for PFS 


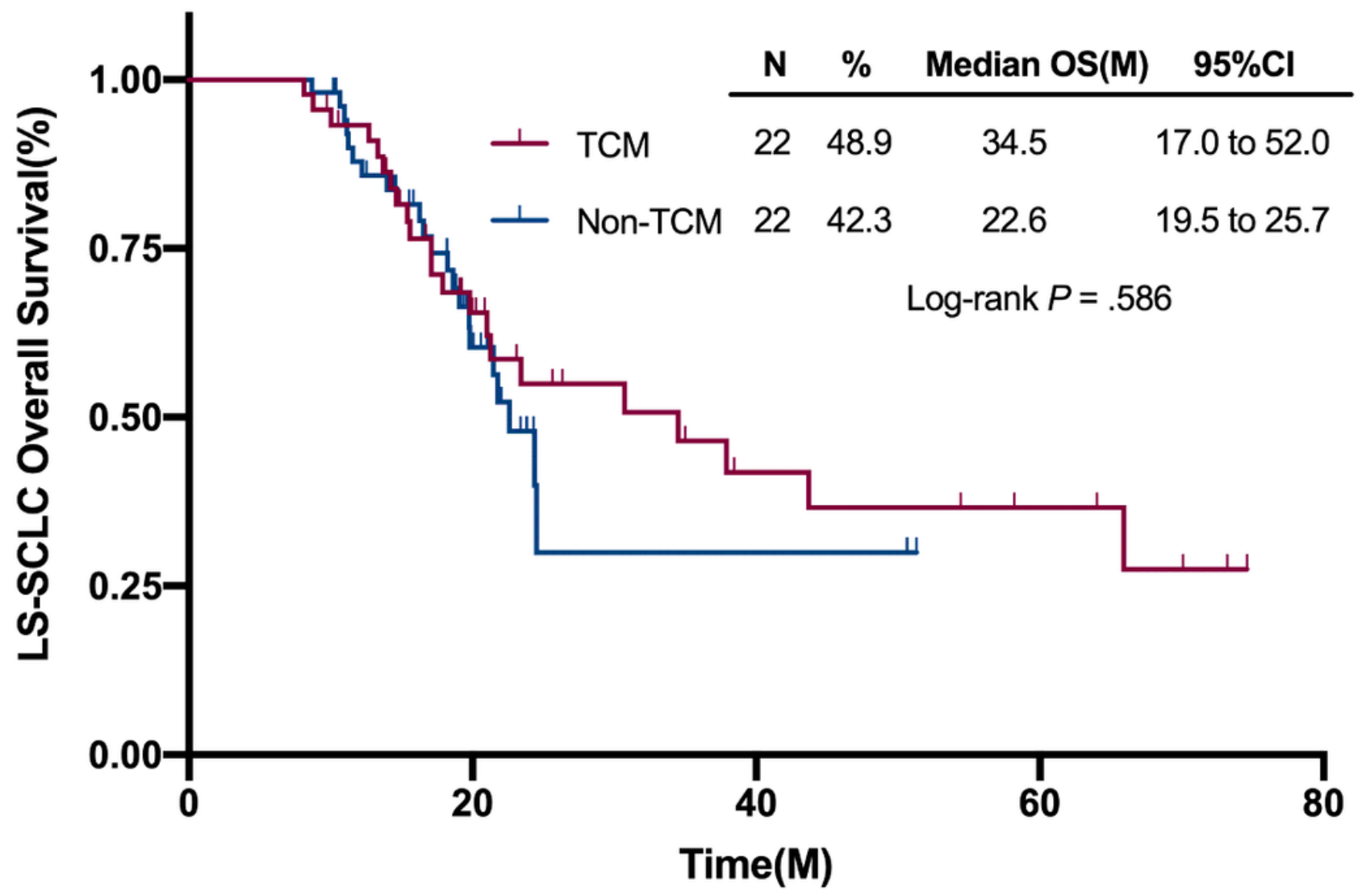

Figure 5

Survival curves of TCM for LSSCLC in OS 


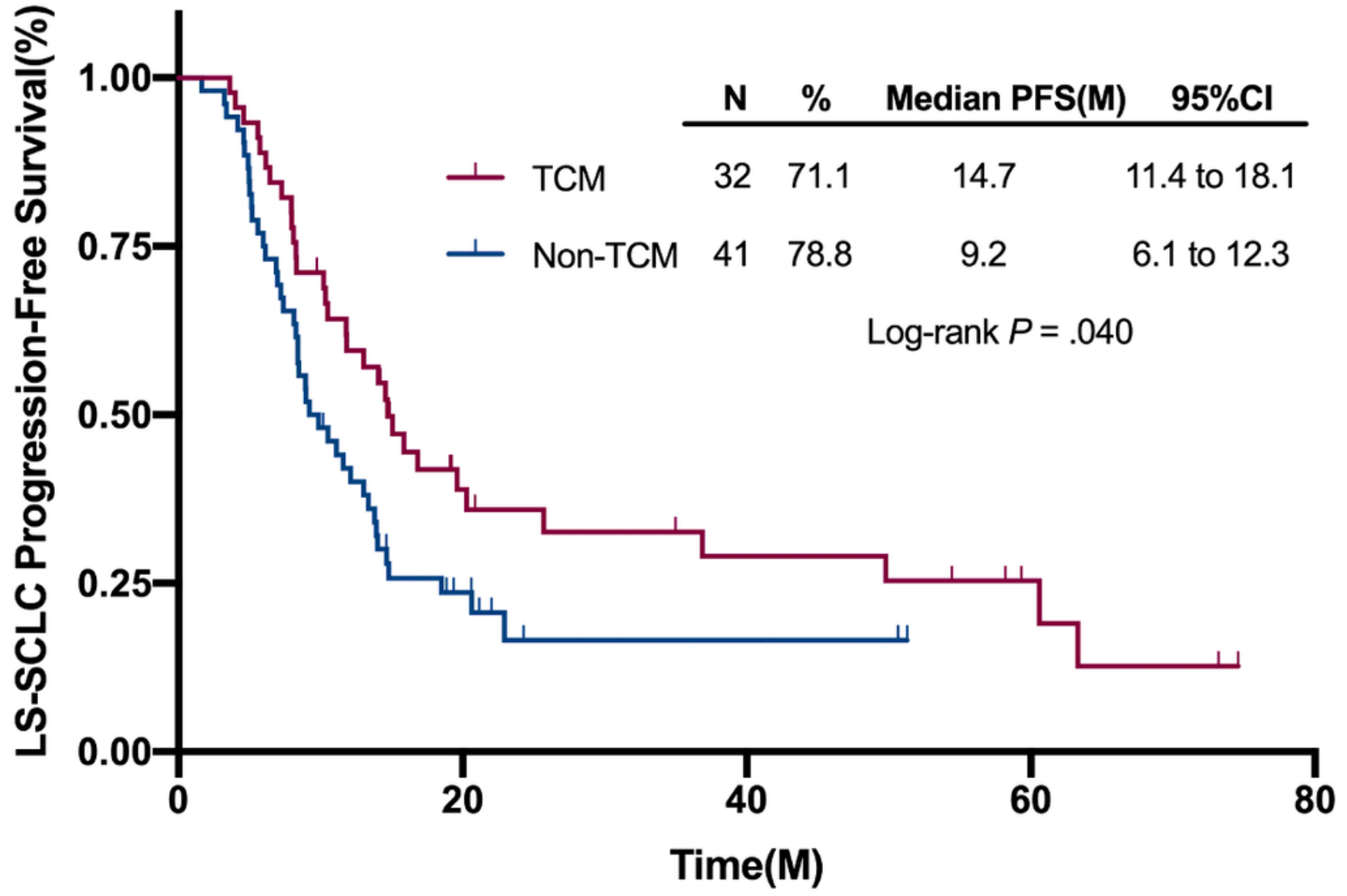

Figure 6

Survival curves of TCM for LSSCLC in PFS 


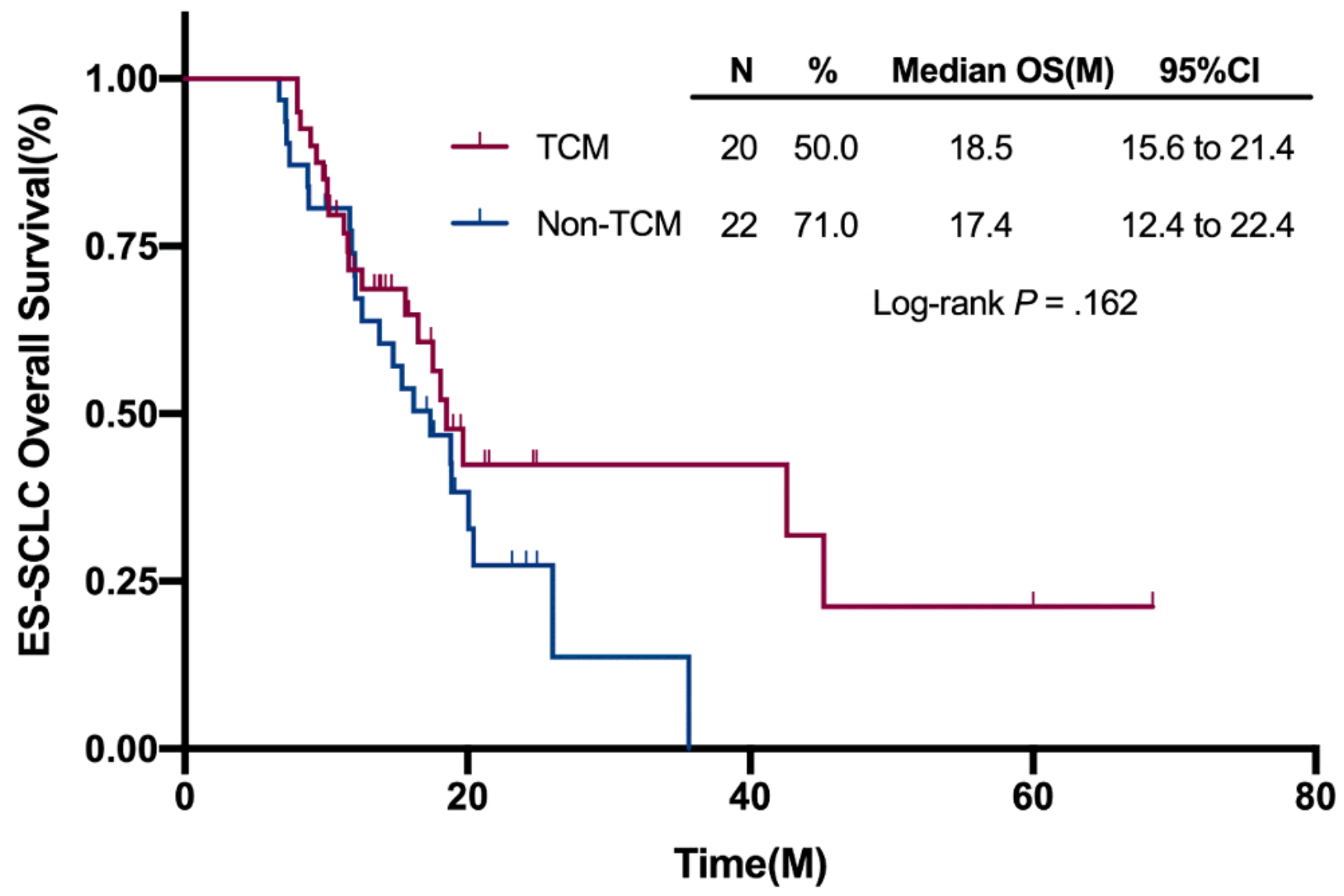

Figure 7

Survival curves of TCM for ESSCLC in OS 


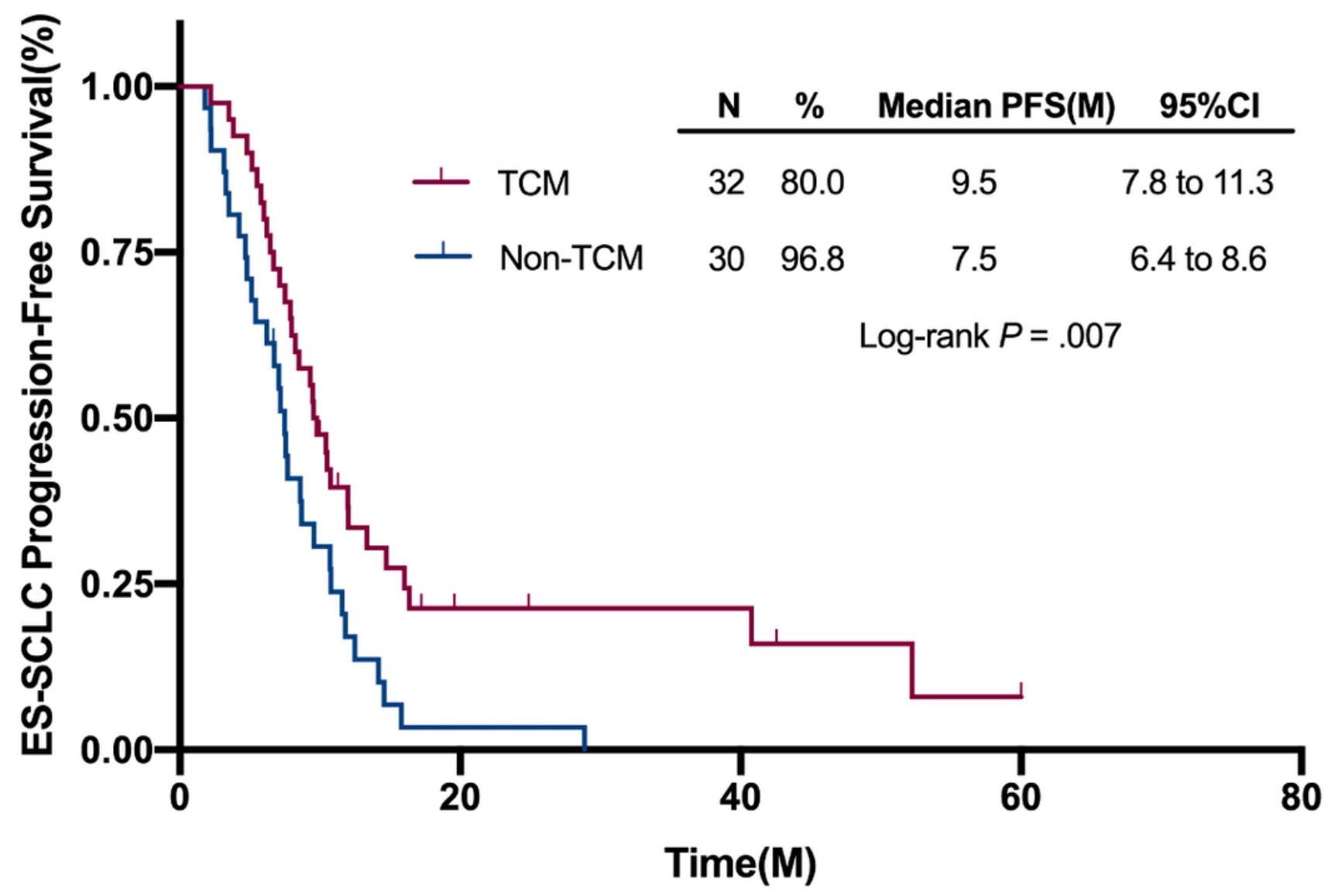

Figure 8

Survival curves of TCM for ESSCLC in PFS 